\title{
Specific Energy Absorption Improvement of Rear Crash Attenuator by Numerical Modelling for Various Angles of Impact
}

\section{NAVEEN VENUGOPAL, ARCHIT MILIND DESHPANDE and HAMID DALIR}

\begin{abstract}
This research ${ }^{*}$ aims on developing a reliable finite element framework to investigate the Specific Energy Absorption (SEA) of the rear crash attenuator of an open-wheel type Indycar vehicle. A meshed model representing the crash structure was designed and its failure behaviour was learnt on the basis of various non-linear finite element modelling techniques to simulate a crash as per regulations from the governing body of Indycar. All the numerical analysis was performed utilizing the LS-DYNA software with the Progressive Failure Model (PFM) and Continuum Damage Model (CDM) of MAT058_LAMINATED_COMPOSITE_FABRIC card.

The sandwich structure material characterization for the tuning of the material model was done by the means of a correlation with experimental data and adjusting the non-physical input parameters in the software. Post calibration, the development of the rear impact attenuator was performed with the model. A combined failure mode was observed with a gradual crushing phenomenon during the analysis on head-on impacts $\left(0^{\circ}\right)$ while in case of oblique impacts performed at $30^{\circ}$ off axis shows the structure failing at its rear attachment points to the bulkhead.

The specific energy absorption was determined at different configurations of impact of this reinforced sandwich structure by evaluating the force over a crushed displacement. The layup was adjusted, the sensitive points at the attachments were stiffened, and the core thickness was varied throughout the structure to improve the overall specific energy absorption by $27.8 \%$ with a gradual deceleration value to that of the prescribed. Finally, the results were compared to the previous Indycar structure and the rear crash attenuator was redesigned with highlights of the refreshed results.
\end{abstract}

Naveen Venugopal, Archit M. Deshpande, Hamid Dalir, Advanced Composite Structures Engineering Laboratory (ACSEL), Purdue School of Engineering and Technology, $799 \mathrm{~W}$ Michigan St, Indianapolis, Indiana, 46202, U.S.A

This is the author's manuscript of the work published in final edited form as:

Venugopal, N., Deshpande, A. M., \& Dalir, H. (2019). Specific Energy Absorption Improvement of Rear Crash Attenuator by Numerical Modelling for Various Angles of Impact. Proceedings of the American Society for Composites - Thirty-Fourth Technical Conference. https://doi.org/10.12783/asc34/31272 


\section{INTRODUCTION}

Every vehicle is designed with the prime objective of safe transportation of its occupants. But when we deal with high speed in lightweight structures specifically in open wheel type race cars, generally known a formula type car, the importance of these structures is immense. These structures of the chassis are known as the crash attenuator and they are involved in absorption of the impact energy during an event of a crash. Over the years, the design of these structures has evolved based on the outer aerodynamic shape of the formula type cars but the engineering underneath that skin defies its limit whenever a crash takes place. Improving its Specific Energy Absorption (SEA) is always the prime target of any composite engineer within the bounds of regulation since that plays a crucial role in the safety of the driver. The application of numerical analysis with the base of Extended Finite Element Methods (XFEM) have made this easier but still, the prediction and approximation in the behaviour of these carbon fiber composite laminates still remain a challenge. This project tries to work more towards solving this challenge by modelling and simulating a rear crash attenuator of an Indycar and validating the results with the experiment to develop a better prototype structure for the future.

A previous study [10-12] conducted on crashworthiness and impact damage of carbon fiber composites via numerical analysis have been following the procedure of using finite element methods and later validating them with experimentation. Every research talk on a different aspect of carbon fiber properties which is crucial from the fact that certain constraints have to be established while we numerically model them using the concepts of continuum damage mechanics. Also, different software is utilized to create such models but the prime focus for such explicit dynamics and non-linear finite element analysis takes place utilizing the software LS-DYNA.

Feraboli [1] talks about how the variation in shape affects the energy absorption of carbon fiber structures during a crash and formulates it based on the curvatures present in the structure through his experimental setup as well as numerical methods. In his research he concludes that in delamination, "little energy is absorbed in splitting the material along an interlaminar crack front" [1]. High energy is absorbed during the fragmentation failure mode and curved geometries suppress delamination fronts and initiates fragmentation, thereby causing higher energy absorption.

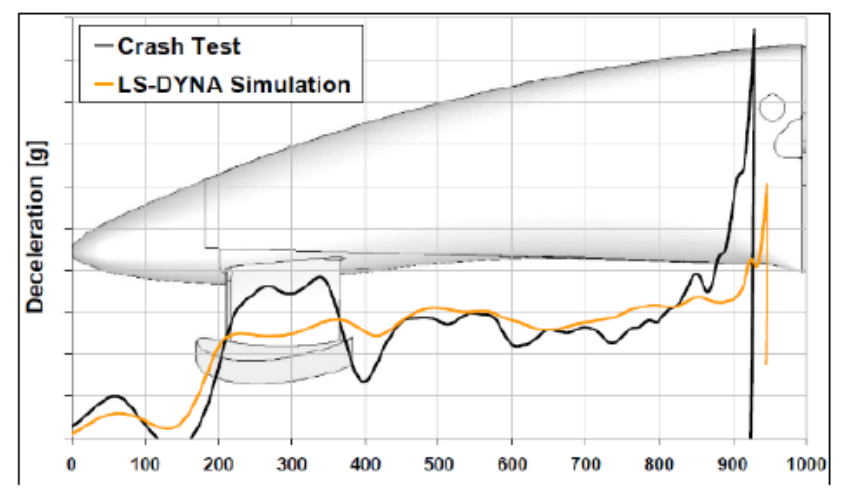

Figure 1. Deceleration-Displacement diagram for frontal impact. 


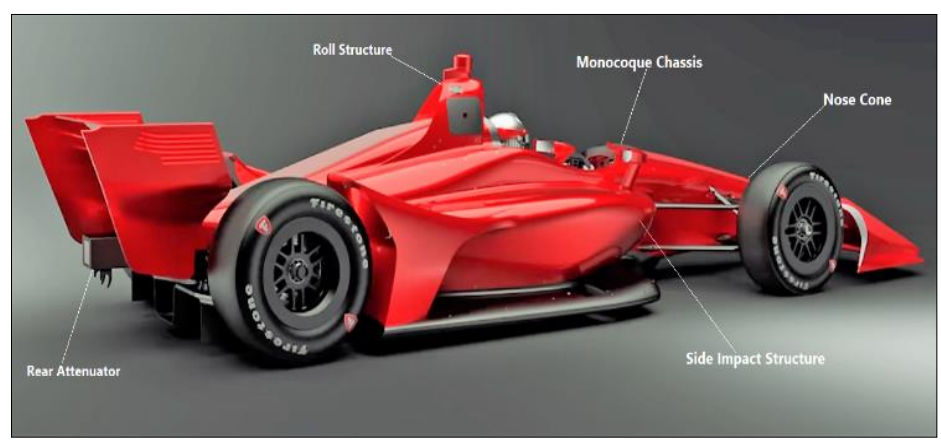

Figure 2. Crash safety structures of 2018 season Indycar.

Agreeing to this concept, Anghileri [2] conducts experiments and find out the deceleration curves using the LS-DYNA where he very well explains the importance and modelling of various parameters in software. He uses MAT_058 card to model the material and provides a sensitivity study on the mesh so that the overall simulation time can be reduced.

Research shows that the energy absorption can be modelled efficiently by utilising chamfer triggers which acts as an intrinsic part of the simulation since it allows a gradual application of force. The use of innovative triggers outperforms the conventional triggers for efficient initiating longitudinal cracking, fragmentation and delamination [4]. The research agrees on the effects of geometrical shape and curvatures as mentioned by Feraboli [1] and discusses the contribution of geometric parameters for effective design.

Cherniav [3] details the important elements of LS-DYNA specifically to model the carbon fiber composites and discusses the shortcomings of three specific material modules and their accuracy with the experiments. He also explains the parameters affecting a simulation and ways to accommodate these in a simulation to get desired results. Wentao [5] explains his investigation on the carbon fibrealuminium sandwich structures by numerically modelling them predicting their energy absorption and improvements by changing the ply direction and thicknesses. While the research focusses on the static drop tests and impact loading, a significant discussion is made on the influence of face sheet thickness on impact resistance and energy absorption in the honeycomb structures. Also, a significant analysis shows that the variation in honeycomb core thickness and cell wall density is an independent factor in the energy absorption during crash [5].

Modelling delamination in a composite structure is tough to achieve and is generally neglected since the amount of energy absorbed in this process is less as compared to splaying and fragmentation. Research by Belingardi [6] shows ways to model this phenomenon of delamination in LS-DYNA using a different material module and doing a comparative study of modelling techniques. Performing this analysis on small specimens and validating it by experiments, he then applicates it to large carbon fiber crash structures. Also, he comments on the "detailed reproduction of the physical phenomenon" [6] at the expense of very high computational time.

Although every author has some specific detail to talk about based on their research and experimentation, they unanimously agree on the future scope and development of carbon fiber structures exploiting its energy absorbing properties which will help in creating safe and crashworthy structures for application in 
motorsports and aerospace. The results produced by Feraboli [1] is validated and approved by the Federal Aviation Administration (FAA) utilized in the design of modern-day aerospace structures. The results produced by Anghileri [2] is utilised by Fédération Internationale de l'Automobile (FIA) for validating their motorsports structures, numerically analyzing them, and further developing it for improved results and designing prototypes.

\section{PROBLEMS AND OBJECTIVES}

The rear impact attenuator of Indycar broke off from its bulkhead at the time of an oblique impact over a series of 3 seasons and three similar crash scenarios taking place at Texas in 2018, and at Iowa in 2017 and 2016. The investigation conducted by the regulatory body of Indycar found that the attenuator breaks off when impact takes place at a $30^{\circ}$ axis or more. Also, in case of a straight impact, the structure does not deform much, causing reduction in energy absorption and car deceleration. This violates the safety of the driver as well as that of other cars during a race.

The objective of this paper is to increase the specific energy absorption of the structure with an optimization analysis on the structural weight by result validation and composite ply optimization. Also, reasonable modification as per the regulation body's requirement specifically the deceleration values of $20 \mathrm{~g}$ will be made to the crash attenuator and improving its performance by redesigning a prototype for the Indycar-2021 season.

The other objectives include developing a better correlation of the modelled structure numerically and understand the working of a composite structure. This will ensure ease in designing and help in reducing the experimental cost analysis.
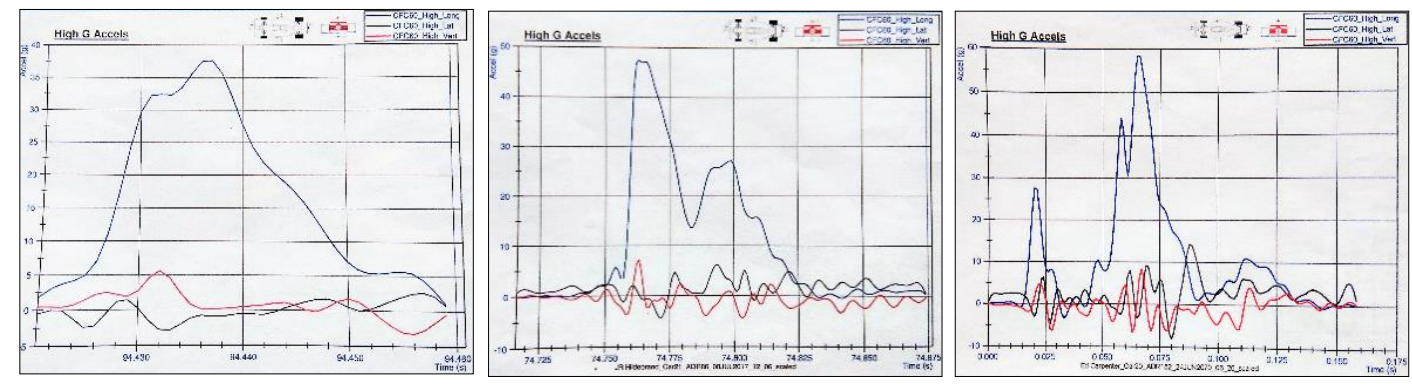

Figure 3. Deceleration-Time plots of the three crash scenarios from the Accident Data Recorder (ADR) in the cars.

(Figure 3.1. Texas 2018 Crash, Figure 3.2,3.3. Iowa 2017, 2016 Crash).

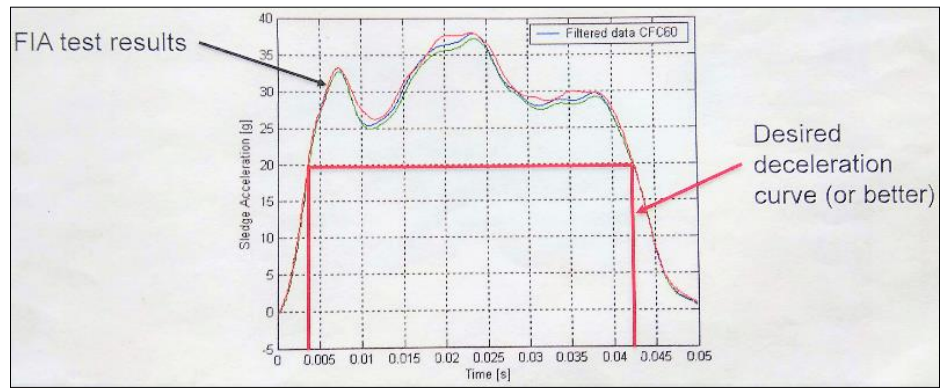

Figure 4. Deceleration vs. Time plot of the experimental crash test conducted by FIA for Indycar. 


\section{MODEL FORMULATION AND EXPERIMENTAL COMPARISON}

\section{Material and Experimental Detail}

The material specifications and the design of the attenuator were provided by the OEM Dallara Automobili. The structural construction of the attenuator includes a carbon fiber-aluminium honeycomb sandwich structure. The skin composed of SC110T2 40\% 3K T300 2x2 twill carbon fiber and the aluminium honeycomb 5052 $1 / 84.5$ pcf for the core. Standard tests as per the FIA guidelines [9] were performed on the attenuator which was accepted by the Indycar for overall design. This includes

- An impactor mass of $900 \mathrm{~kg}$ impacting at a speed of $11.9 \mathrm{~m} / \mathrm{s}$.

- The peak deceleration over the first $225 \mathrm{~mm}$ of deformation does not exceed $20 \mathrm{~g}$.

- The maximum deceleration does not exceed $20 \mathrm{~g}$ for more than a cumulative $15 \mathrm{~ms}$, this being measured only in the direction of impact.

- All the structural damage must be contained within the area behind the rear wheel centre line [9].

The impact test was performed, and the deceleration graphs were plotted which certainly shows the need for remodelling of the structure since the peaks overshoot the design limits (Fig. 4) causing hindrance to safety.

To understand the working of this specific carbon fiber prepreg and its equivalent mesh modelling in LS-DYNA, square tube specimens were tested under axial compression load with a $45^{\circ}$ chamfer made of this material. The layup for the testing was chosen to be $[0,45]_{\mathrm{s}}$ with a ply thickness of $0.25 \mathrm{~mm}$. The tests were conducted on MTS-858 Mini Bionix II and the samples were crushed to over $60 \%$. The data output was plotted as force vs. displacement curves which were later used for the result validation in LS-DYNA.
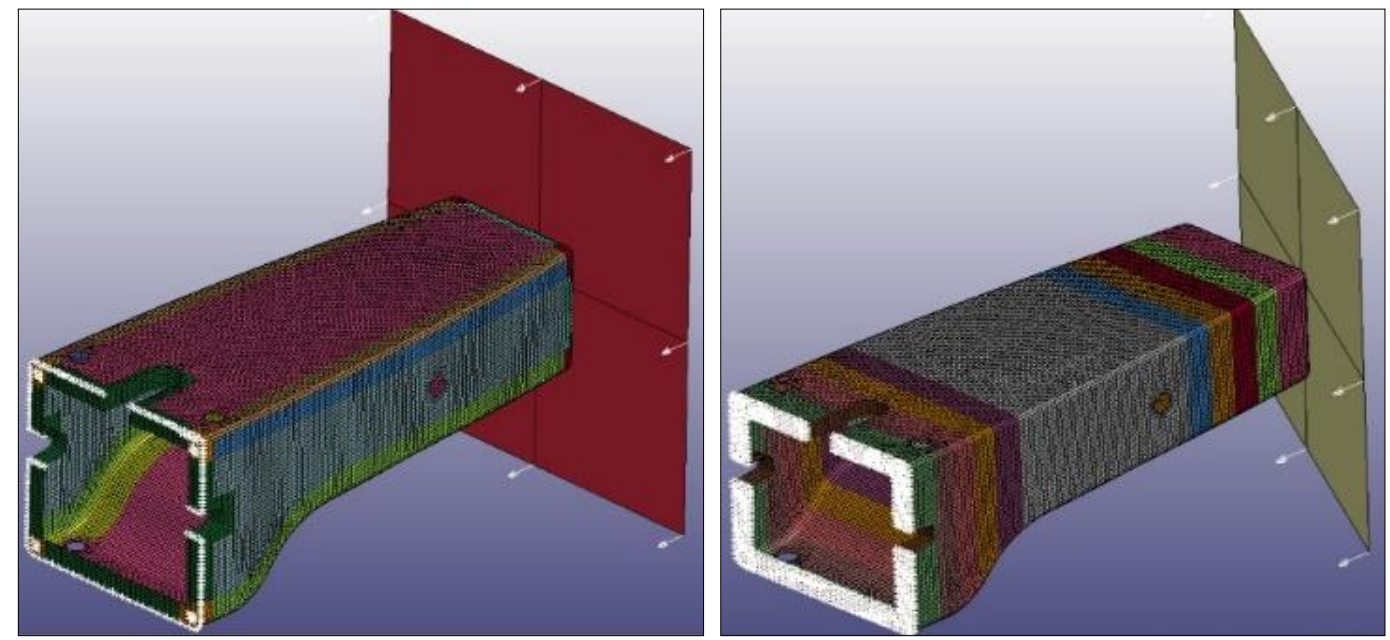

Figure. 5. Finite Element Model for the crash simulation as per the FIA Regulation at $0^{\circ}$ and $30^{\circ}$ off axis. 


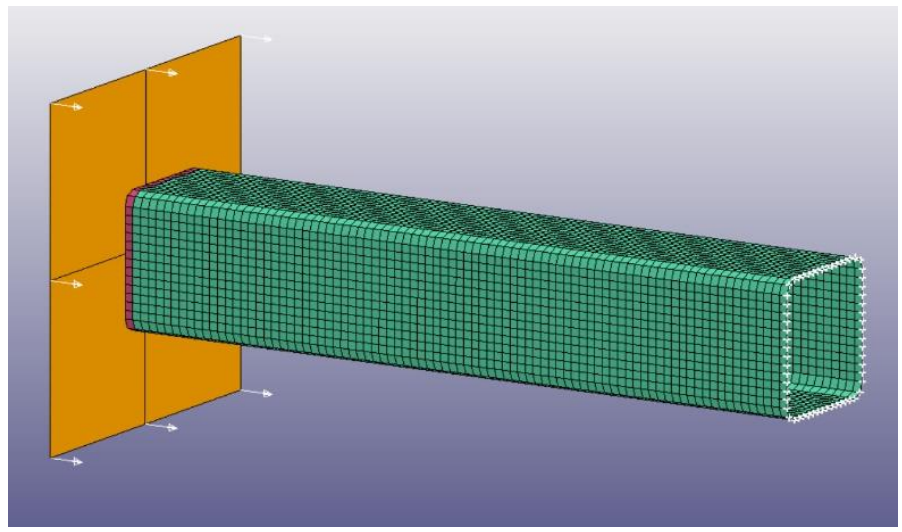

Figure 6. Specimen model simulation for calibration of the LS-DYNA software.

\section{Finite Element Material Modelling}

MAT058_LAMINATED_COMPOSITE_FABRIC module in LS-DYNA was utilised to model for the numerical simulation. This is a Matzenmiller's damage mechanics-based model accounting for non-linear peak softening of the composite plies [7]. This is inferred as two sets of parametric properties Ei and SLIMi which consists of the physically defined material properties and individual non-physical parameters which needs to be calibrated as per the experimental data. The table illustrates the parametric data that was used for calibration of the software to establish an experimental correlation and baseline for the development simulation runs. The MAT058 material model consists of two parts to determine the element erosion- the first being the damage mechanics model that involves modulus reduction using three non-negative parameters which vary from 0 to 1 [4]. The other part is the complete removal of element layer after maximum effective strain is achieved using ERODS.

The specimen model was meshed with Belytschko-Tsay thin shell elements and the trigger was modelled as an element wide consisting of half the number of plies in the overall structure. The impactor was designed as a rigid body and the structural force was measured using the RC_FORCE card. The parametric properties of SLIMi were adjusted to calibrate the software for the further runs. The validation with the experiments ensured these values to be within the limits for effective simulation.

The rear attenuator CFRP outer skin was meshed using the quality optimization tool included in the HyperMesh software. This tool is a Quality Index based tool, which optimizes the mesh following a chosen algorithm and a variety of parameters as per designer's choice. The crash analysis algorithm was utilised here which performs the geometric simplifications and places the elements in a fashion that the crashworthiness analysis is optimized. This CFRP skin was assigned with the Belytschko-Tsay thin shell elements [7] for analysis and the number of integration points increase with the increase in addition of layers using the 
PART_COMPOSITE module of LS-DYNA. A total of 28673 elements were identified and the boundary constrains similar to that of the experimental setup was designed using BOUNDARY_SPC. The impactor was modelled as a rigid body [2] with a weight of $900 \mathrm{~kg}$ and was constrained to move in only $\mathrm{x}$-direction with other motion fixed at a velocity of $11.9 \mathrm{~m} / \mathrm{s}$. The simulation was run on 16 core $3.3 \mathrm{GHz}$ AMD Ryzen computer and the total time for simulation was about 8 hours and 43 minutes. The graphs of deceleration, forces and displacement were obtained in the form of Binary_D3 plots.

\section{PARAMETRIC ANALYSIS ON FAILURE CRITERIA}

\section{Deformation \& Failure Mechanics}

The energy dissipation in metallic materials takes place through "plastic folding, work hardening and adiabatic losses due to heating" [13]. Contrastingly, carbon fibers "dissipate energy through external and internal frictions, fiber bending and kinematic dissipation through fragmentation" [13]. In addition, one of its properties of high specific energy absorption has been exploited to achieve vehicle deceleration during crash. During a brittle fracture in composites, 4 of the energy absorbing phenomena could take place- global buckling, progressive folding, progressive splaying and progressive fragmentation. The damage mechanism may occur in four different ways in a fiber reinforced plastic. This includes fiber failure, matrix failure, fiber-matrix shear failure and delamination [14].

Fragmentation dominated the energy absorption mechanism resulting in energy absorption of over $50 \mathrm{~kJ}$. Also, Mode-1a failure which is failing of the structure by micro-cracks was observed as the failure mode in the rear attenuator structure. Delamination was not modelled specifically due to very high simulation times and its comparatively less involvement in energy absorption over the other failure model. Since matrix failure is the dominant cause of delamination, the amount of energy absorbed by the matrix over the fibers is usually $10 \%$. Also, since the structure has high amount of curvatures, the delamination failure is suppressed [1].

\section{Effect of Ply Thickness}

Thinner unidirectional plies are usually used in aerospace grade manufacturing but the trend in motorsports has been a bit different. Though a material with a specified thickness was provided by the manufacturer, a study was conducted to understand the effect of ply thickness on the deceleration values which is one of the prime objectives of the research. Four different plies of thicknesses ranging from $0.135 \mathrm{~mm}$ to $1.08 \mathrm{~mm}$ were simulated on the test specimen with the experimentally determined parametric properties. It is to be noted that the overall thickness of the structure was kept same while simulating the results which can be inferred as the variation in the number of plies involved in the structure (Table I) 
TABLE I. STUDY OF PLY THICKNESS AND ITS EFFECT ON PEAK

\begin{tabular}{cccc}
\hline Ply Thickness (in mm) & Number of Plies & Peak Deceleration (in g) \\
\hline 0.135 & 32 & 20.1 \\
0.27 & 16 & 17.2 \\
0.54 & 8 & 13.9 \\
1.08 & 4 & 11.2 \\
\hline & & & \\
\hline & & & \\
\hline
\end{tabular}

Figure 7. Force $(\mathrm{N})$ vs Displacement $(\mathrm{mm})$ Plot to find the total energy absorbed by the attenuator (Figure 7.1. Current vs Figure 7.2. Redesigned).
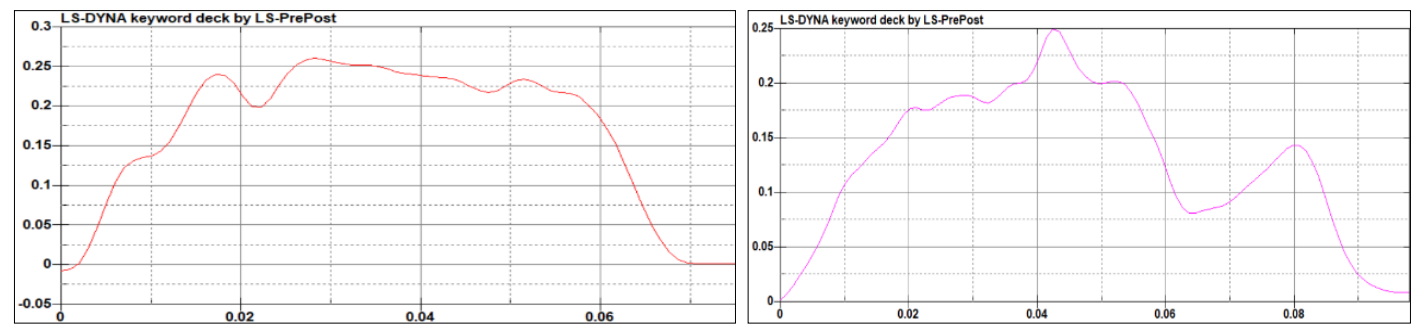

Figure 8. Deceleration $(\mathrm{x} 100 \mathrm{~g})$ vs. Time (s) plot of the simulated result for the redesigned carbon fiber layup at $0^{\circ}$ and $30^{\circ}$ angle of impact.

\section{Effect of Impact Angles}

The impacts taking place during a race is rarely head-on and the attenuator has to be designed in a way so that it sufficiently absorbs the torque produced in case of an oblique impact rather than shearing off from its attachment. Deceleration and energy absorption at two different impact angles of $15^{\circ}$ and $30^{\circ}$ of axis were measured to study its effects. In both the simulations performed using the calibrated LS-DYNA, we found the peak deceleration to be $30 \mathrm{~g}$ and $25 \mathrm{~g}$ lasting for less than 2 milliseconds which is under the norms of FIA regulations [9]. While the $15^{\circ}$ impact represented a deceleration curve close to $0^{\circ}$ head-on impact but lasted for over $50 \%$ more and reflected higher SEA. The impact at $30^{\circ}$ also lasted for $50 \%$ more than the $0^{\circ}$ head-on impact but reflected a trough which can be evaluated as the failure of bulkhead taking place at that time. This can be adjusted to get better values since the amount of energy absorbed closely matches to the $0^{\circ}$ head-on impact and can be justified for the bulkhead failure. 


\section{RESULTS AND DISCUSSIONS}

The specific energy absorption was determined at different configurations of impact of the rear attenuator. This was achieved by evaluating the force over a crushed displacement of the reinforced sandwich structure. The layup was adjusted, the sensitive points were stiffened, and the core thickness was varied throughout the structure to improve the overall SEA by $27.8 \%$ with a gradual deceleration value to that of the prescribed. This overall design worked well for both the head-on impacts and angular impacts as required by the governing body of Indycar and are well under the aforementioned mentioned regulations.

The redesigned rear attenuator is capable of absorbing 58\% more energy and is optimised for minimum weight improving it by $34.5 \%$. The further modification of the structure for changing its overall design was limited to the prototype phase since this may influence the aerodynamics of the car. The validation of the redesigned attenuator awaits experimental correlation, but since the baseline results have a 90\% match with the experimental data, the improved results obtained via numerical modelling and simulations are precise.

\section{REFERENCES}

[1] Feraboli. P., Wade. B., "Crushing Behavior of Laminated Composite Structural Elements: Experiment and LS-DYNA Simulation", DOT/FAA/TC-15/25, Federal Aviation Administration, Washington, DC, December 2016.

[2] Anghileri. M., Benetton. D., Bassi. S.M., "Numerical Modelling of a Crash-absorbing Nosecone", Matr. 876746, Dipartimento di Scienze e Tecnologie Aerospaziali, Politecnico di Milano, June 2018.

[3] Cherniaev. A., Montesano. J., Butcher. C., "Modelling the Axial Crush Response of CFRP Tubes using MAT054, MAT058 and MAT262 in LS-DYNA", 15th International LS-DYNA Users Conference, June 2018.

[4] Tong. Y., Xu. Y., "Improvement of Crash Energy Absorption of 2D Braided Composite Tubes Through an Innovative Chamfer External Triggers", International Journal of Impact Engineering, vol. 111, pp. 11-20, January 2018.

[5] He. W., Yao. L., Meng. X., "Effect of structural parameters on low-velocity impact behaviour of aluminium honeycomb sandwich structures with CFRP face sheets", Thin Walled Structures, vol. 137, pp. 411-432, April 2019.

[6] Belingardi. G., Obradovic. J., Boria.S., "Experimental and numerical investigation of the impact behavior of composite frontal crash structures" Composite Part b, vol. 79, pp. 20-27, September 2015.

[7] Shi. D., Xiao. X., “An Enhanced Continuum Damage Mechanics Model for Crash Simulation of Composites”, Composite Structures, vol. 185, pp. 774-785, February 2018.

[8] Melo. D. R. B., Cunha. F. S. R. P., Lau. F. J. P., "Composite Structures Impact Simulation Behaviour", Department of Aerospace Engineering, Instituto Superior Técnico, Lisboa, November 2014.

[9] FIA, "2019 Formula One Technical Regulations”, vol 0, pp. 81-83, March 2019.

[10] Thornton. P.H., "The crush behavior of pultruded tubes at high strain rates", Journal of Composite Materials, vol. 24, pp.594, July 1990.

[11]Hull. D., "A unified approach to progressive crushing of fibre reinforced composite tubes", Composites Science and Technology, vol. 40, pp. 377-421, January 1991.

[12] Boria. S., Forasassi. G., "Honeycomb sandwich material modelling for dynamic simulations of a crash-box for a racing car”, WIT Transactions on the Built Environment, vol. 98, pp. 167-176, September 2008.

[13]Elmarakbi. A., "Automotive Composite Structures for Crashworthiness", ISBN 9781118423868, First Edition, October 2013. 
[14] Salas. P., Venkataraman. S., "Controlling failure using structural fuses for predictable progressive failure of composite laminates", Structures and Multidisciplinary Optimization, vol. 34. pp. 473-489, December 2007. 